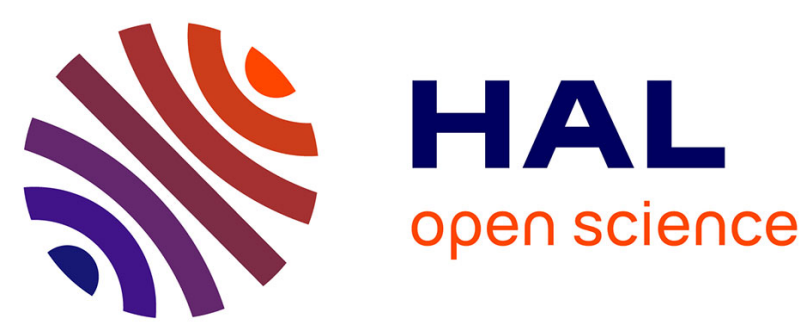

\title{
Benchmarking smartphone performances for cooperative disaster alert diffusion
}

Farouk Mezghani, Nathalie Mitton

\section{To cite this version:}

Farouk Mezghani, Nathalie Mitton. Benchmarking smartphone performances for cooperative disaster alert diffusion. AdHoc-Now 2018 - 17th International Conference on Ad Hoc Networks and Wireless, Sep 2018, Saint Malo, France. hal-01826642

\section{HAL Id: hal-01826642 \\ https://hal.inria.fr/hal-01826642}

Submitted on 29 Jun 2018

HAL is a multi-disciplinary open access archive for the deposit and dissemination of scientific research documents, whether they are published or not. The documents may come from teaching and research institutions in France or abroad, or from public or private research centers.
L'archive ouverte pluridisciplinaire $\mathbf{H A L}$, est destinée au dépôt et à la diffusion de documents scientifiques de niveau recherche, publiés ou non, émanant des établissements d'enseignement et de recherche français ou étrangers, des laboratoires publics ou privés. 


\title{
Benchmarking smartphone performances for cooperative disaster alert diffusion
}

\author{
Farouk Mezghani and Nathalie Mitton \\ Inria Lille \\ \{farouk.mezghani, nathalie.mitton\}@inria.fr
}

\begin{abstract}
When a disaster strikes, communication infrastructure such as cellular network may get destroyed, which makes rescue operations more challenging. Short range-based opportunistic communications using daily mobile devices such as smartphones present a promising solution to support infrastructure failure. In a previous work, we have proposed COPE, a cooperative opportunistic alert diffusion solution useful for trapped survivors during disasters to ease and speed up their rescue and assistance. It targets to maintain mobile devices alive as long as possible for a maximum network coverage until reaching proximate rescuers. COPE leverage mobile devices that come with multiple network technologies and aims to perform a systematic network interface selection. We have implemented a proof-of-concept of COPE for android smartphones using two network technologies: Bluetooth and Wi-Fi. This work presents a benchmark analysis of performances of smartphones based on COPE. Testing experiments have been carried out to measure the performance of smartphones in terms of energy consumption, clock synchronization and transmission range. We believe that such experimental results can support technological choices for rescue operations but also for many other applications relying on smartphone performances.
\end{abstract}

\section{Introduction}

Opportunistic communications have attracted considerable attention as a possible alternative to support infrastructure failure during disaster scenarios $[5,6]$. Indeed, mobile devices used daily by everyone such as smartphones can be useful to offer direct communications helpful to ease rescue operations during disasters such as to report location position of trapped survivors after earthquake.

Several research works have proposed solutions for disaster recovery leveraging mobile devices. However, these latter have left behind two important features: (i) multi-network assortment and (ii) various energy levels. These works have considered mobile devices that come with only one network technology while nowadays, mobile devices such as smartphones are equipped with multiple network technologies (e.g. Wi-Fi, Bluetooth and cellular) and users have no idea which is the best. Moreover, they do not consider mobile devices that come with various initial energy levels which obviously has an impact on the disaster recovery solution.

Unlike existing works, in a previous work, we have proposed a cooperative opportunistic alert diffusion solution named COPE useful for survivors during 
disasters to report their location, thus, to speed up their rescue operation [4]. COPE leverages multiple network technologies and considers mobile devices that come with various initial energy levels. It aims to maintain a maximum network coverage by keeping mobile devices alive for as long as possible. We have implemented a proof-of-concept of COPE for android smartphones considering Bluetooth and Wi-Fi technologies [3]. An overview of COPE solution is presented in the next section.

The contribution of this paper lies in a benchmark analysis of performances of smartphones considering COPE. We have conducted testing experiments and we provide results showing the performance of smartphones in terms of energy consumption, clock synchronization and transmission range. These results can also serve to support technological choices for rescue operations but also for many other applications relying on smartphone performances.

\section{Background : The COPE mechanism}

Fig. 1 illustrates a multi-technology communication view of COPE considering mobile devices equipped each with three network technologies $\left(n_{1}, n_{2}\right.$ and $\left.n_{3}\right)$ corresponding for instance to those available nowadays in smartphones (Bluetooth, Wi-Fi, Cellular). This work classifies the available network technologies according to their energy consumption (E) and transmission range (TR) as follows: $T R_{n_{3}}>T R_{n_{2}}>T R_{n_{1}}$ and $E C_{n_{3}}>E C_{n_{2}}>E C_{n_{1}}$.

e.g. Bluetooth provides the shortest transmission range, thus consuming the lowest energy amount. This work relies on the assumption that network technologies can be sorted from the lowest to the higher energy-consuming and that there is a direct correlation between energy consumption and network performances (especially in terms of range). One of the goals of this paper is to validate or invalidate this assumption.

Nodes exchange periodic beacon messages to form cliques based on the less power network technology $n_{1}$ (i.e. cliques $C_{i}, \mathrm{i}=1 . .5$ ). Inside each clique, a cooperative communication is performed based on the $n_{2}$ network technology. Indeed, inside each clique, nodes alternately diffuse the alert message and discover neighbors using the network technology $n_{2}$. Time is divided into equal time-slots $\tau$ and each node determines its wake-up schedule based on the clique information (IDs and energy levels of nodes belonging to the same clique). The wake-up order is determined based on the node ID in comparison to those of nodes belonging to the same clique (i.e. node with the lowest ID occupies the first period during the time-slot). During its wake-up, each node activates its network interface $n_{2}$ for neighboring discovery and alert diffusion, otherwise, it switches to the power save mode.

If a node $i$ discovers other proximate nodes $j$ from the $n_{2}$ network perspective (nodes $i$ and $j$ are neighbors with $n_{2}$ technology), together, they form a zone that includes their respective cliques (the zone is a clique at an upper level). For instance, as shown in Fig. 1-Layer $n_{2}$ communication, nodes $s_{10}$ and $s_{13}$ discover each other based on the $n_{2}$ network interface. Then, they exchange information 


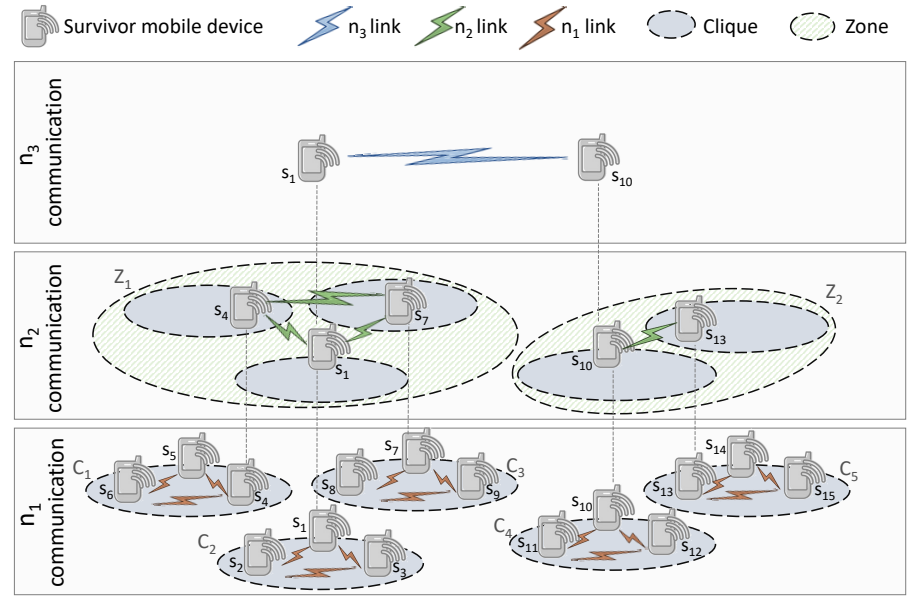

Fig. 1. Multi-technology communication overview

(i.e. nodes IDs and energy levels) about their corresponding cliques $C_{4}$ and $C_{5}$ and then form a zone $Z_{2}$ that includes their corresponding cliques $C_{4}$ and $C_{5}$. Afterwards, they diffuse the zone information (nodes belonging to the zone and their energy levels) to their cliques through the active interface $n_{1}$.

Inside each zone, a cooperative diffusion can be performed from the $n_{3}$ communication perspective based on an alternative alert diffusion. Each node computes its wake-up schedule from the $n_{3}$ communication perspective by referring to its energy level and ID and those of nodes inside the same zone.

The network topology is dynamic due to leaving and joining nodes. This latter can be detected through the periodic messages exchanged between nodes belonging to the same clique/zone. When the topology changes, nodes exchange their 1-hop neighbors and update their cliques information and re-computes their wake-up schedules.

\section{Smartphone performance}

We have conducted different experiments featuring six smartphones Wiko Tommy 2 and exploiting two network technologies Bluetooth and Wi-Fi. The main specifications of used smartphones are shown in Table 1. Testing scenarios target to measure and evaluate the performances of the smartphones using COPE application and considering the energy consumption, clock synchronization and transmission range metrics.

\subsection{Energy consumption}

COPE should be energy efficient in order to maintain mobile devices alive as long as possible since rescue operations might take long time. Testing scenarios have been conducted to measure the energy consumption considering three network topologies; (i) one node operates individually, (ii) two nodes cooperate based on COPE, and (iii) three nodes cooperate based on COPE. Measurements have 
Table 1. Smartphones main specifications

\begin{tabular}{ll}
\hline Smartphone model & Wiko Tommy 2 \\
\hline OS & Android 7.1 (Nougat) \\
Battery & Li-Po 2500 mAh 9.5 Wh \\
Bluetooth & 4.1, A2DP, LE \\
WiFi & Wi-Fi Direct \\
\hline
\end{tabular}

been carried out by only running COPE application (i.e. no other applications running in parallel) and the smartphone screen is turned off. Fig. 2 illustrates the energy consumption over five minutes from the WiFi and Bluetooth perspective considering the three network topologies. Results show that when a node operates individually, it consumes more energy than in the other topologies where nodes are cooperating. Moreover, as we increase the number of nodes within the group, the energy consumption is reduced since nodes will be in a sleep mode for a longer period from the WiFi perspective. Therefore, cooperative scheme can help to reduce the energy consumption and thus to keep mobile devices alive for a longer time.

We would like to emphasize that these experiments validate that Bluetooth consumes less energy than WiFi in the context of applications requiring an exchange of small data (e.g. short text message). In the context of COPE that only needs to send short SOS messages, this confirms our assumptions.

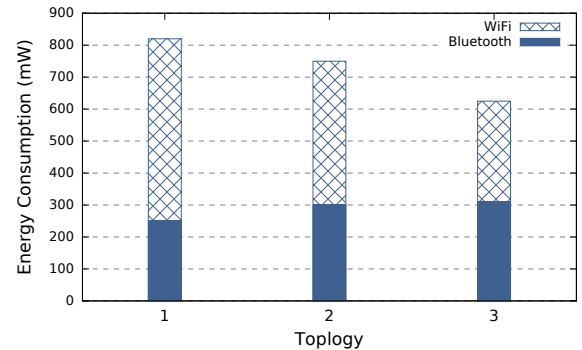

Fig. 2. Energy consumption

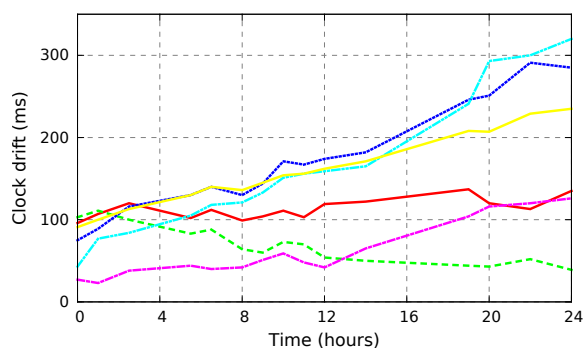

Fig. 3. Clock drift of six smartphones

\subsection{Clock drift}

COPE assumes that mobile nodes are already synchronized since smartphones get the local time from the network providers with millisecond accuracy before disasters occur. In the following, we study the clock drift of the smartphones to check weather an additional synchronization is required during the post-disaster period. We have carried out a first simple experiment to check weather smartphones belonging to different network operators are synchronized. Results have shown that smartphones are a few milliseconds apart. Next, we have conducted an experiment to test the clock drift featuring the six smartphones. We initially synchronized all the mobile phones through Internet via a NTP (Network Time 
Protocol) time server. Afterwards, we prevent the automatic synchronization and we measure the clock drift referring to the NTP time server.

Fig. 3 shows the clock drift of the different smartphones during a period of 24 hours. Results show that mobile phones desynchronize by up to $0.3 \mathrm{~s}$ during 1 day which is not significant drift and does not impact the COPE scheme. Therefore, since the time-slot $\tau$ is at second level, COPE does not require an additional synchronization. We would like to emphasize that we have repeated the experiment considering various scenarios: smartphone display ON/OFF, smartphone in charge/not in charge and by running applications in parallel. We have obtained similar clock drift results. Smartphones synchronize their clock time with the cellular infrastructure. When disconnected from the cellular network, clock drift is not significant while difficult to predict. Indeed, results show that smartphones present different desynchronization behaviors even though we use the same mobile device model (i.e. Wiko Tommry 2).

\subsection{Transmission range}

We have carried out experiments to test the transmission ranges of Bluetooth and WiFi-Direct. Testing the quality of the link and the transmission speed are not in the scope of this work. Indeed, we consider the necessity of exchanging short text messages that can be useful for alerting or asking for assistance. Thus, in our testing scenarios, we simply try to exchange some short messages of few bytes between smartphones and we keep increasing the distance between mobile nodes until the link interruption. We have carried out various testing scenarios considering: windy and humid weathers; calm and dry weathers; outdoor line of sight (see yellow lines on Fig. 4); indoor with obstacles (1 to 2 walls); between two buildings with a distance of about $80 \mathrm{~m}$ (see red line on Fig. 4).

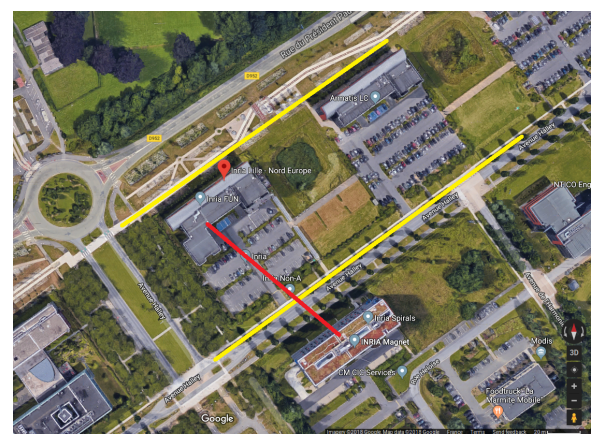

Table 2. Bluetooth and Wi-Fi

Direct transmission range

\begin{tabular}{lll}
\hline & Bluetooth & Wi-Fi Direct \\
\hline Indoor & $\sim 35 \mathrm{~m}$ & $\geq 100 \mathrm{~m}$ \\
Outdoor & $\sim 50 \mathrm{~m}$ & $\geq 100 \mathrm{~m}$ \\
\hline
\end{tabular}

Fig. 4. Transmission range testing area

Table 3.3 presents the transmission range of the Bluetooth and Wi-Fi Direct for outdoor and indoor scenarios. These results show the importance of the new version of Bluetooth offering an important transmission range comparing to the previous versions. Several research works consider the transmission range of Bluetooth of around $10 \mathrm{~m}$ while the new version of Bluetooth (Bluetooth Low 
Energy BLE) offers more important transmission ranges compared to what is expected even in theory. Moreover, experiments validate the assumptions considered in COPE solution that WiFi offers a higher transmission range than Bluetooth.

\section{Related works}

Some research works have studied the performance of smartphones mainly in terms of energy consumption. Work in [2] have evaluated the energy consumption of wireless technologies (Bluetooth, WiFi, and $3 \mathrm{G}$ ) that can be useful to reduce energy consumption for collaborative downloading. Authors in [1] evaluated the energy consumption based of smartphones based on its different components (e.g. CPU, displays). For data communication, most of these studies target to evaluate the performances of smartphones based on simple data exchange without referring to a specific application. In this work, we provide an evaluation of smartphone based on energy consumption and transmission range useful as reference for applications based on a short data communication. Additionally, we give an evaluation of clock synchronization between a group of smartphones.

\section{Conclusion}

This work investigates the smartphones behavior for opportunistic alert diffusion during disaster scenarios. It gives a benchmark analysis of performances of smartphones and provides additional experimental results of performances of COPE. Experiments have been conducted and results show that COPE can reduce energy consumption comparing to the individual alert diffusion. Moreover, testing experiments have been conducted showing results of clock synchronization and transmission range of Bluetooth and Wi-Fi that can facilitate deep study of advances for rescue operations and also for many other applications relying on smartphone performances. Adding another form of network technology (e.g. on the fly cellular network) to the proof-of-concept implementation and evaluating it in terms of energy consumption is a subject of our ongoing work.

\section{References}

1. Carroll, A., Heiser, G., et al.: An analysis of power consumption in a smartphone. USENIX annual technical conference 14, 21-21 (2010)

2. Kalic, G., Bojic, I., Kusek, M.: Energy consumption in android phones when using wireless communication technologies. MIPRO, 2012 Proceedings of the 35th international convention pp. 754-759 (2012)

3. Mezghani, F., Bardol, V., Mitton, N., Laporte, J.L.: An android application for opportunistic alert diffusion in disaster scenario. IEEE PIMRC 2017 (2017)

4. Mezghani, F., Mitton, N.: Alternative opportunistic alert diffusion to support infrastructure failure during disasters. Sensors 17(10), 2370 (2017)

5. Nishiyama, H., Ito, M., Kato, N.: Relay-by-smartphone: realizing multihop deviceto-device communications. IEEE Communications Magazine 52(4), 56-65 (2014)

6. Trifunovic, S., Kouyoumdjieva, S.T., Distl, B., Pajevic, L., Karlsson, G., Plattner, B.: A decade of research in opportunistic networks: challenges, relevance, and future directions. IEEE Communications Magazine 55(1), 168-173 (2017) 